\title{
UVB radiation induces an increase in intracellular zinc in human epidermal keratinocytes
}

\author{
CHRISTIAN J. STORK ${ }^{1}$, LISA M. MARTORANO ${ }^{2}$ and YANG V. LI ${ }^{1,2}$ \\ ${ }^{1}$ Molecular and Cellular Biology Program, ${ }^{2}$ Department of Biomedical Science, \\ Ohio University, Athens, OH 45701, USA
}

Received December 28, 2009; Accepted February 22, 2010

DOI: 10.3892/ijmm_00000486

\begin{abstract}
Ultraviolet (UV) radiation is known to cause oxidative stress, inflammation, DNA damage and apoptotic cell death; however, many details of these malign mechanism have yet to be elucidated. In this study, the exposure of adult human epidermal keratinocytes (HEKa) with UVB (>100 mJ/ $\mathrm{cm}^{2}$ ) resulted in the significant increase of intracellular zinc that was released from its storage and was detected by fluorescent zinc indicators. Toxicity testing revealed that UVB-induced zinc release in HEKa is associated with HEKa cell death. Cells that showed elevated intracellular zinc fluorescence upon UVB exposure were also stained by propidium iodide (PI), a traditional viability indicator whose fluorescent signal is as a result of its intercalating with DNA fragments and is unaffected by zinc concentration, showing significant colocalization [Pearson's correlation coefficients $r=0.956(\mathrm{n}=6)]$. The cytotoxicity of zinc was also determined by an MTT assay after applying the exogenous zinc $\left(\mathrm{ZnCl}_{2}\right)$ along with its ionophore pyrithione $(20 \mu \mathrm{M})$ into HEKa culture medium. A significant reduction in cell viability as a function of both zinc concentration and exposure time was observed. The treatments of 1,10 and $100 \mu \mathrm{M} \mathrm{ZnCl}_{2}$ with pyrithione demonstrated 2.3, 60 and $84 \%$ cell deaths, respectively (control $0.5 \%)$ after $30 \mathrm{~min} . \mathrm{ZnCl}_{2}(100 \mu \mathrm{M})$ was also found to induce complete HEKa death after $1 \mathrm{~h}$. Thus, the present study demonstrates that UVB irradiation-induced increased zinc is detrimental to HEKa viability, and zinc may be a necessary step in UVB-induced cell death signaling pathways.
\end{abstract}

\section{Introduction}

Zinc is an abundant transition metal ion in cells, and is an important structural and functional component in many cellular

Correspondence to: Dr Yang V. Li, Department of Biomedical Sciences, Ohio University, 346 Irvine Hall, Athens, OH 45701, USA

E-mail: li@oucom.ohiou.edu

Key words: zinc, cell death, ultraviolet, keratinocytes, skin, cytotoxicity, Newport Green proteins and enzymes $(1,2)$, such as in zinc finger transcription factors and metallothioneins $(3,4)$. Zinc is normally tightly regulated, limiting the extent of intracellular labile (free) zinc concentrations $\left(\left[\mathrm{Zn}^{2+}\right]_{\mathrm{i}}\right)(2,5)$; therefore, an excessive increase in zinc is generally believed to contribute to cellular demise $(6,7)$. Several lines of evidence indicate that oxidative and nitrosative stress lead to an increase of intracellular zinc and that zinc cytotoxicity may, in turn, stimulate and interfere with vital signaling pathways (8-10). This zinc cytotoxicity proceeds with observable signs of necrosis such as cell body swelling and destruction of intracellular organelles as well as signs of apoptosis such as DNA fragmentation and caspase activation $(7,8)$.

Ultraviolet (UV) light has been recognized as the major environmental causative factor in skin cell damage. Particularly, the ultraviolet B (UVB) irradiation of human skin is known to induce pathophysiological processes such as oxidative stress and inflammation, and is the most prominent risk factor for the development of skin cancer (11-13). Increasing evidence supports the role of oxidative stress in cancer development (14-16). The UV response in skin is a multifaceted biological process with complex signaling pathways that ultimately determine the fate of the skin cells $(12,17,18)$. Although the precise molecular mechanism underlying these events remains elusive, an increasing body of evidence indicates that detrimental UV irradiation triggers oxidative stress, DNA damage, and apoptotic cell death (11,19-23). Therefore, understanding the molecular mechanisms that underlie the process of apoptotic cell death in UV exposed keratinocytes will help decipher the pathological basis of clinical skin disorders including skin cancer and will facilitate the development of novel therapeutic strategies.

While UVB is a pro-oxidative stressor with profound effects on skin (24-26), the action of zinc in UVB-induced cell death is largely unknown. Zinc homeostasis is affected by oxidative stress, which is suggested to be a potent trigger for detrimental zinc release from its intracellular storage $(10,27,29)$. In this study, we investigated the potential dysregulation of zinc homeostasis using human epidermal keratinocytes (HEKa) exposed to UVB radiation. HEKa were preloaded with a fluorescent zinc indicator to evaluate the change of intracellular zinc after UVB irradiation. Our study showed that UVB irradiation evoked an increase of intracellular zinc. Staining with a cell viability indicator revealed 
that the increasing zinc or zinc accumulation in HEKa was associated with UVB-induced cell injury/death. The increase in $\left[\mathrm{Zn}^{2+}\right]_{\mathrm{i}}$ via the application of exogenous zinc reduced HEKa viability as a function of both zinc concentration as well as exposure time, representing a novel detrimental factor in the viability of skin cells. Thus, the UVB irradiationinduced zinc overload may be a necessary step in UVB induced cell death signaling pathways.

\section{Materials and methods}

Materials. Newport Green AM, FluoZin-3 AM, MTT [3-(4,5dimethylthiazol-2-yl)-2,5-diphenyltetrazolium bromide], TPEN [tetrakis-(2-pyridylmethyl)ethylenediamine], 1,10phenanthroline and propidium iodide were purchased from Invitrogen (Carlsbad, CA, USA). Pyrithione (1-Hydroxy-2pyridinethione sodium salt), and buffer salts were purchased from Sigma (St. Louis, MO, USA).

HEKa culture. HEKa cells and growth medium were purchased from Invitrogen and seeded into collagen coated 24-well plates or 35-mm glass bottom petri dishes. Cultured cells were maintained in a humidified incubator with $5 \% \mathrm{CO}_{2}$ at $37^{\circ} \mathrm{C}$, and maintained in Epilife Medium containing Epilife defined growth supplement according to the manufacturer's instructions. Cells were routinely subcultured prior to reaching confluence.

UV irradiation. Direct UVB irradiation of cultured HEKa cells was employed as follows: Cells grown in 35-mm glass bottom petri dishes were mounted on the stage of a fluorescence microscope with a UV-light source (8 watts, $3 \mathrm{UV}^{\text {тм }}$ lamp, UVP, Upland, CA) suspended above the stage. This arrangement of experimental setup avoided potential artifacts by motion, and enabled continuous fluorescence acquisition before and after the irradiation. UVB intensity in this experimental setup was monitored (average UVB intensity $=7 \mathrm{~mW} / \mathrm{cm}^{2}$ ) and UVB exposure times were employed so as to achieve the UVB dosages indicated in the text.

Intracellular zinc measurement. All imaging experiments were performed in HEPES medium containing the following (in $\mathrm{mM}$ ): $130 \mathrm{NaCl}, 5 \mathrm{KCl}, 8 \mathrm{MgSO}_{4}, 1 \mathrm{Na}_{2} \mathrm{HPO}_{4}, 25$ glucose, 20 HEPES, 1 Na-Pyruvate; pH adjusted to 7.4 (HEPES medium). Cultured cells were rinsed 3 times with PBS and then were loaded with either Newport Green AM $(10 \mu \mathrm{M})$ or FluoZin-3 AM $(10 \mu \mathrm{M})$ for $30 \mathrm{~min}$ at $37^{\circ} \mathrm{C}$. After dye loading, cells were rinsed 3 times with $\mathrm{PBS}$ and equilibrated to room temperature for $30 \mathrm{~min}$ prior to use. Fluorescence was monitored using a customer-designed inverted fluorescence microscope equipped with a mercury lamp light source. Images were processed and analyzed using Image-Pro or NIH ImageJ software. Cells were monitored using a 488/15-nm BP filter for excitation and a 530-nm LP emission filter.

\section{Viability assays}

Propidium iodide (PI) assay. Following experimental treatments, the viability of HEKa cells was analyzed by PI assay. PI is excluded from entering the lipid bilayer because of the molecule size and charge, but in nonviable cells, the dye freely enters the damaged membrane to bind nucleic acids (DNA and RNA) and yield bright red fluorescence. In this study, PI was used at a final concentration of $10 \mu \mathrm{g} / \mathrm{ml}$ in HEPES medium. Cells were incubated in PI solution for $30 \mathrm{~min}$ at room temperature and PI uptake was measured using an inverted fluorescent microscope with a mercury lamp light source. PI uptake was monitored using a 543/20 nm BP excitation filter and a $580 \mathrm{~nm}$ LP emission filter.

Colocalization analysis. Colocalization experiments were conducted using a Zeiss LSM 510 (confocal) microscope as previously described (30). Cultured cells were examined using a Plan-Neofluar 10x or 40x objective. Cells were subjected to the indicated UVB dosages, and then switched to perfusate containing the following reagents, which were applied at the final concentration dissolved in HEPES medium. The extracellular form of Newport Green (hexapotassium salt) was used at a final concentration of $10 \mu \mathrm{M}$ and was imaged with excitation by a 488-nm laser line and a BP505-530 emission filter. PI was used at $10 \mu \mathrm{g} / \mathrm{ml}$ with excitation by a $543-\mathrm{nm}$ laser line and a BP560-615 emission filter. The two fluorescence channels were scanned sequentially to avoid crosstalk between signals. Images were processed and analyzed using Zeiss software. The colocalization of red and green image pairs was measured and the Pearson's correlation coefficient was used to assess statistical significance where $\mathrm{p} \leq 0.05$ was considered significant.

Cytotoxicity (MTT assay). The MTT assay is based on the production of purple formazan pigment from methyltetrazolium salt by the mitochondrial enzymes of viable cells. The color density is proportional to mitochondrial enzyme activity, reflecting the number of viable HEKa. Therefore, the MTT assay is sensitive to the function of active mitochondrial enzymes, which typically lose activity early in the progression towards death. After exposure to the indicated experimental treatments, the viability of HEKa cells grown in 24-well plates was analyzed by MTT assay. Cells were rinsed 3 times with PBS, equilibrated for $30 \mathrm{~min}$ at $37^{\circ} \mathrm{C}$, then were incubated for $4 \mathrm{~h}$ at $37^{\circ} \mathrm{C}$ in PBS containing MTT at a concentration of $1 \mathrm{mg} / \mathrm{ml}$. Following incubation the MTT solution was aspirated, wells were rinsed three times with PBS, and the deposited formazan crystals were solubilized in DMSO. The absorbance of the MTT was measured at $570 \mathrm{~nm}$ using a BioMate 3 spectrophotometer (Thermo Scientific, Rochester NY). The viability of cells in each treatment group ( 8 wells per group) was expressed as a percentage of the control group. Significant differences between groups were evaluated by a one-way analysis of variance (ANOVA) where $p \leq 0.05$ was considered significant.

\section{Results}

UVB irradiation induces an increase of intracellular zinc in $H E K a$. To measure the intracellular zinc after UVB irradiation, HEKa were preloaded with the fluorescent zinc indicators. Cells seeded in petri dish were then placed and held on microscope stage for UV exposure and fluorescence measurement. This arrangement of experimental setup avoided potential artifacts by motion, and enabled continuous fluorescence acquisition before and after the irradiation. Data in Fig. 1 

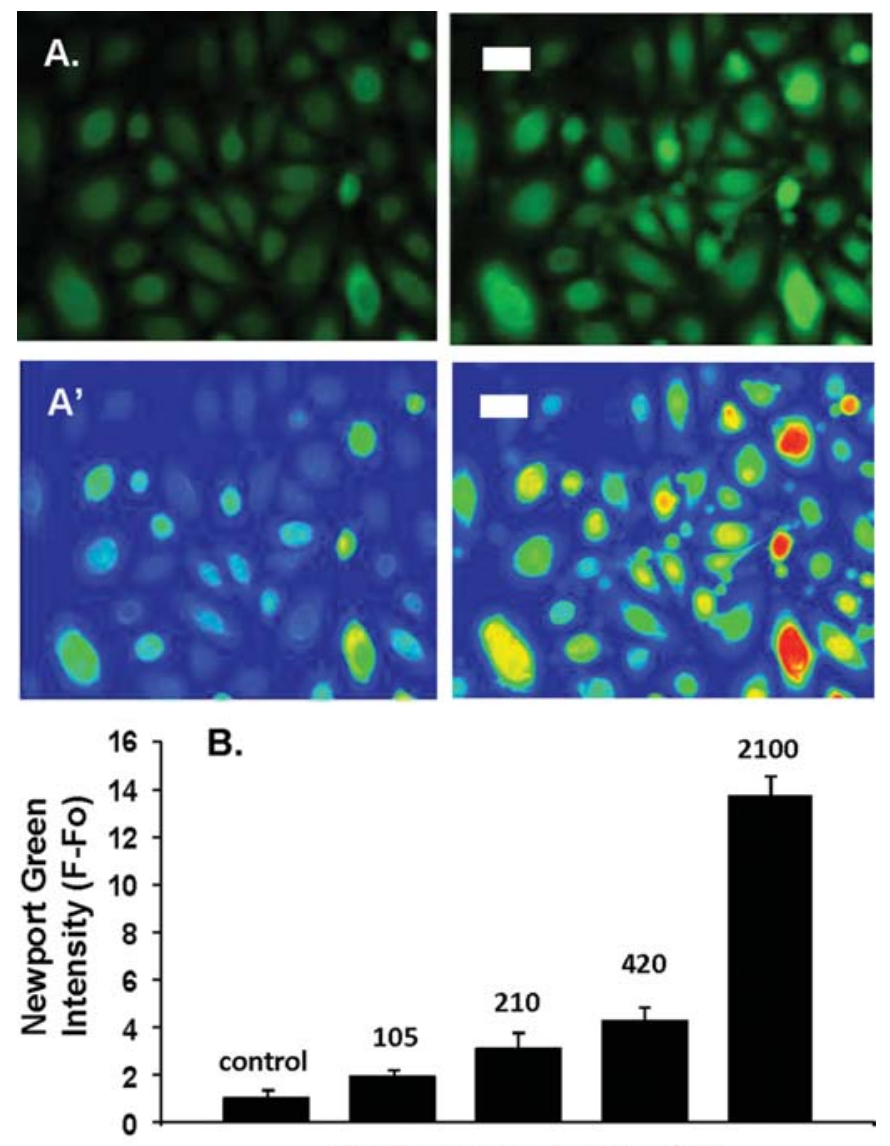

B.

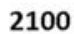

UV Exposure $\left(\mathrm{mJ} / \mathrm{cm}^{\wedge} 2\right)$

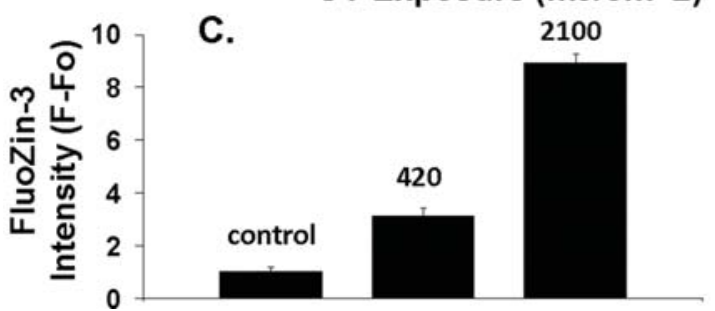

Figure 1. Increase of intracellular zinc measured with fluorescent zinc indicator Newport Green and FluoZin-3. (A) Fluorescent images of HEKa preloaded with Newport Green and viewed at $\mathrm{x} 40$. The left was green fluorescence taken before UVB irradiation, and the right was taken after UVB irradiation. UVB intensity was $2,100 \mathrm{~mJ} / \mathrm{cm}^{2}$. Scale bar $=25 \mu \mathrm{m}$. (A') is the same as (A) but with a pseudocolor scale. (B) Dose-dependent increases of intracellular zinc measured with Newport Green after UVB irradiation. Data are means $\pm S E, n=6$. (C) UVB induced dose-dependent zinc increase measured with another fluorescent zinc indicator Fluozin-3. Data are means $\pm S E, n=5$. The fluorescence $(F)$ is normalized to the baseline fluorescence $\left(\mathrm{F}_{0}\right)$.

were acquired in the cells preloaded with fluorescent zinc indicator Newport Green $\left(\mathrm{K}_{\mathrm{D} \text { zinc }}=1 \mu \mathrm{M}, 10 \mu \mathrm{M}\right)$ and show that, after the irradiation, there was a substantial increase in intracellular zinc concentration. The increase in intracellular zinc was detected immediately after UVB irradiation, and was dose-dependent with the irradiation (Fig. 1B). The change of zinc fluorescence was observed after only $15 \mathrm{sec}$ $\left(105 \mathrm{~mJ} / \mathrm{cm}^{2}\right)$ irradiation, and higher fluorescent intensity was measured after longer irradiation. The UVB dose-dependent increase appeared to reach peak with $5 \min \left(2,100 \mathrm{~mJ} / \mathrm{cm}^{2}\right)$ for Newport Green loaded cells. The increase in intracellular zinc by UVB irradiation was verified with another fluorescent zinc indicator FluoZin-3 $\left(\mathrm{K}_{\mathrm{D} \text { zinc }}=15 \mathrm{nM}, 10 \mu \mathrm{M}\right)$. As in Newport Green the increase was also dose-dependent with UVB irradiation recorded in HEKa preloaded with FluoZin-3 (Fig. 1C). Higher fluorescent intensity was measured after longer irradiation. Based on the affinity of Newport Green $\left(\mathrm{K}_{\mathrm{D} \text { zinc }}=1 \mu \mathrm{M}\right)$ to zinc and the range of its fluorescence detection previously described (31), the maximum amount of accumulated zinc released by UVB irradiation was estimated to be $\sim 10 \mu \mathrm{M}$.

UVB induces rising $\left[\mathrm{Zn}^{2+}\right]_{i}$ and cell death. The accumulation of intracellular zinc has been implicated to be a detrimental factor associated with cell injury/death (6-10). Next, we determined whether UVB induced zinc increase was associated with cell injury or cell death. In this study, cell viability after the radiation was comparatively determined with dual staining of propidium iodide (PI), a conventional marker for cell injury, and Newport Green, a fluorescent zinc indicator, in HEKa. As expected, extensive UVB irradiation $\left(3,150 \mathrm{~mJ} / \mathrm{cm}^{2}\right)$ induced an accumulation of intracellular zinc. When viewed using a fluorescence confocal microscope (645-nm filter) after the irradiation (Fig. 2), cells with green Newport Green staining also showed contrasting red PI staining. Therefore, the dual fluorescent staining, or colocalization, of Newport green and PI staining showed agreement between endogenous increases in intracellular zinc and indications of neuronal damage with Pearson's correlation coefficient $r=0.956(n=6)(30,32)$ after UVB irradiation. These data suggested that cells with rising intracellular labile zinc after UV radiation were damaged and became necrotic or were undergoing apoptotic cell death.

Exogenous zinc induces cytotoxicity in HEKa. We then investigated whether zinc can cause HEKa death. If zinc is directly associated with or mediates UVB-induced cell injury/death, increases in intracellular zinc via the application of exogenous zinc will cause the reduction of HEKa viability. In this set of experiments, we treated cells with zinc $\left(\mathrm{ZnCl}_{2}\right)$ and its ionophore (Na-pyrithione) that facilitated the influx of zinc into cells and the subsequent increasing in intracellular zinc (facilitated by co-application of zinc ionophore pyrithione) (33-36). First, cell viability was measured, similar to above, with PI to stain dying or dead cells. HEKa were treated separately with various concentrations $(1,10$, and $100 \mu \mathrm{M})$ of zinc with $30 \mathrm{~min}$ incubation. The increase in intracellular zinc produced concentration-dependent cell death (Fig. 3). The cell death appeared to develop rather quickly; there was an increase in PI positive cells in the treatment of $1 \mu \mathrm{M}$ zinc for $30 \mathrm{~min}$. The treatment with 10 or $100 \mu \mathrm{M}$ zinc caused more than two or three times, respectively, higher PI positive cells than in the control. Total cell death (the positive control) was induced by lysis with $1 \%$ (v/v) Triton X-100. The incubation of cells with $10 \mu \mathrm{M}$ zinc for 30 min produced $\sim 30 \%$ of total death.

Finally, zinc cytotoxicity of HEKa viability was further tested by MTT cell viability assay in separate experiments. Similar to PI assay, the MTT results demonstrated a concentration-dependent cytotoxicity after exposure to zinc for 30 min (Fig. 4). The cytotoxicity was evident at 10 and $100 \mu \mathrm{M}$ zinc applications. The percentage of MTT reduction 

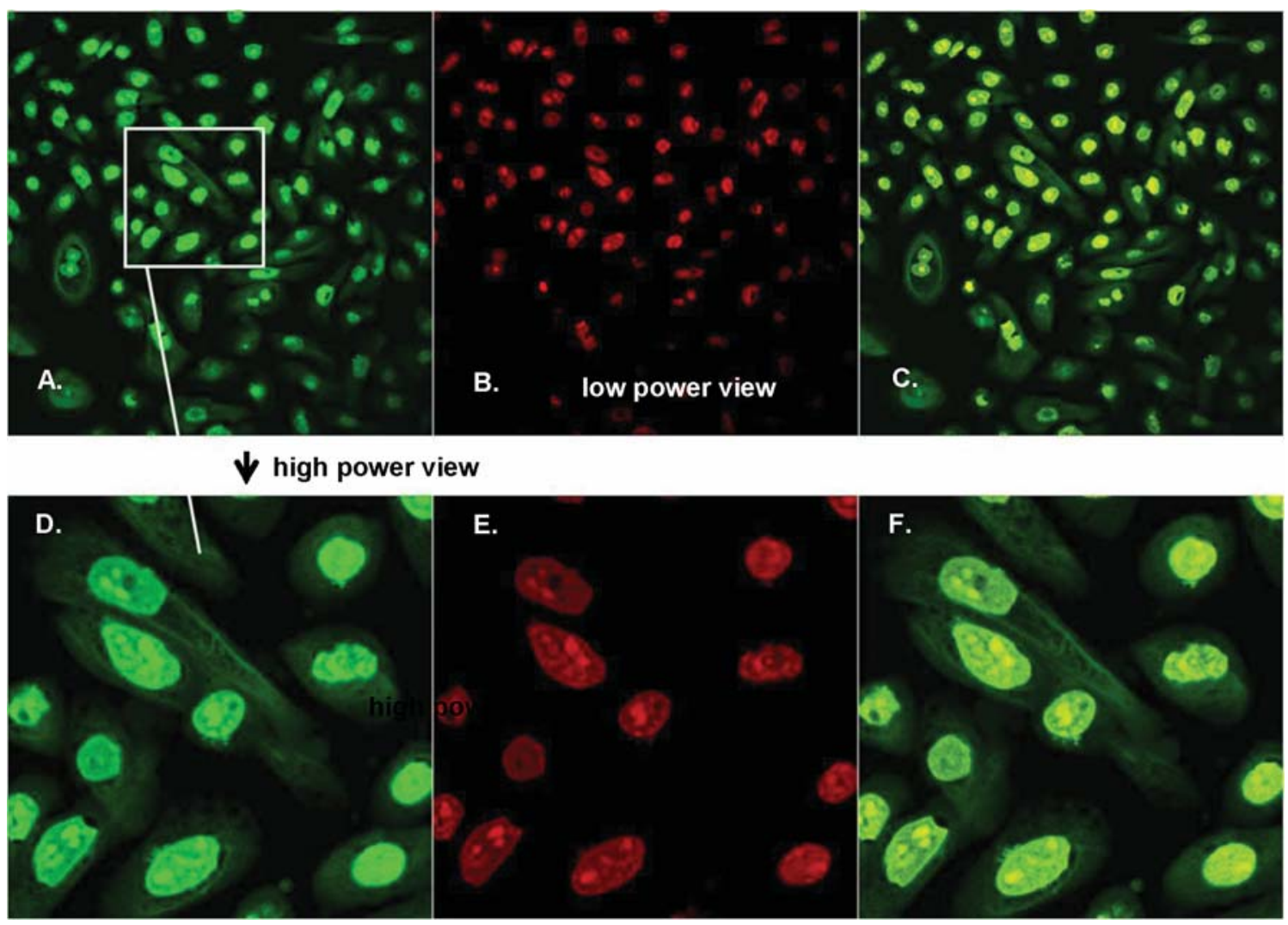

Figure 2. Colocalization study of zinc increase and cell injury/death in HEKa. (A-F) are microscopic fluorescence confocal images of HEKa stained with Newport Green and PI. Colocalization of Newport Green and PI staining was accomplished by doing two channel laser scans, after which Zeiss imaging software produced single channel results and a composite image produced by fluorescent overlay. (A-C) images viewed at x40, and (D-F) the same images but viewed at x100. (A and D) images of Newport Green-stained HEKa green with emission at $505 \mathrm{~nm}$; (B and E) PI-stained red HEKa with emission at $645 \mathrm{~nm}$; (C and F) composites of overlay fluorescence images showing colocalization of Newport Green and PI. Scale bar $=50 \mu \mathrm{m}$ in $(\mathrm{A}-\mathrm{C})$ and $10 \mu \mathrm{M}$ in $(\mathrm{D}-\mathrm{F})$.
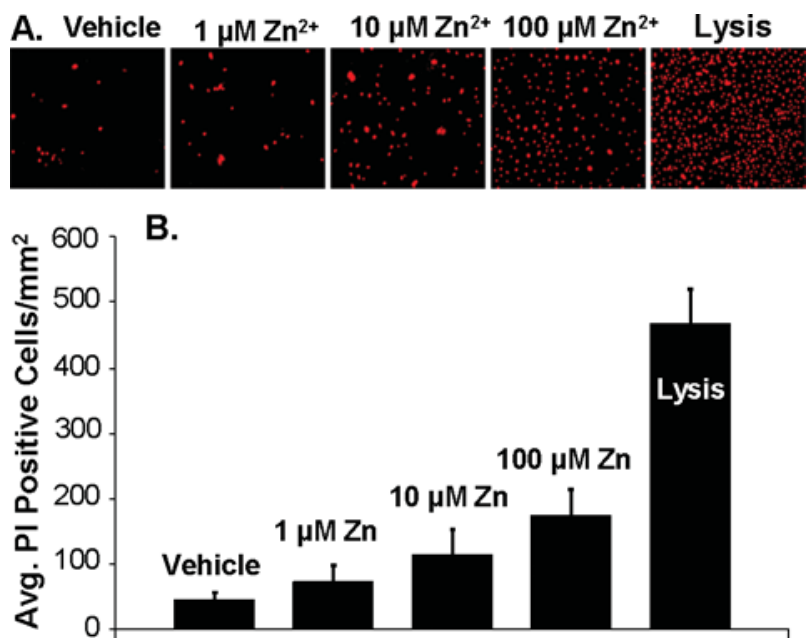

Figure 3. HEKa viability measured by PI after zinc treatments. (A) Representative images of PI positive cells (red fluorescence) analyzed 30 min after addition of zinc $(1,10,100 \mu \mathrm{M})$ and pyrithione $(20 \mu \mathrm{M})$. Total cell death by $1 \%(\mathrm{v} / \mathrm{v})$ Triton X-100 provided positive control. Scale bar $=250 \mu \mathrm{m}$. (B) Graph of the average (means \pm SEM, $n=6$ samples per treatment) number of PI positive HEKa (per $\mathrm{mm}^{2}$ ) after the treatment of various concentrations of zinc for $30 \mathrm{~min}$.

(relative to normal control) observed after $30 \mathrm{~min}$ exposure at concentrations 1,10 and $100 \mu \mathrm{M}$ was 3,61 , and $85 \%$, respectively, with a further reduction to $100 \%$ by lysis with
Triton X-100 (data not shown). The greater toxicity measured by the MTT assay is likely a result of the differences in the viability assays, where the MTT assay largely measures overall mitochondrial reduction potential; which would likely be lost prior to the development of the membrane damage measured by the PI assay. Further, mitochondria have been widely reported to be inhibited by increases in intracellular zinc (37-39).

\section{Discussion}

The major finding in the present work is that the accumulation of intracellular free zinc in HEKa may be a detrimental factor in UVB-induced skin injury or cell death. The present study shows that UV irradiation produces rising zinc levels in cytosolic space (Fig. 1). We demonstrate further that the accumulation of intracellular zinc is colocalized in the same cells that show positive PI staining (Fig. 2) after UV radiation, suggesting that the rising zinc is associated concomitantly with the radiation induced cell death. The possible cytotoxicity of zinc induced by UVB irradiation in HEKa is further supported by the results of exogenous zinc application that directly increases intracellular labile zinc. The applications of zinc directly into media induced the concentration-dependent HEKa injury/death as determined using two viability assays, the PI assay (Fig. 3) and the MTT assay (Fig. 4). Cell viability was reduced and apoptosis was enhanced with increasing zinc concentrations. Thus, the results in the present 


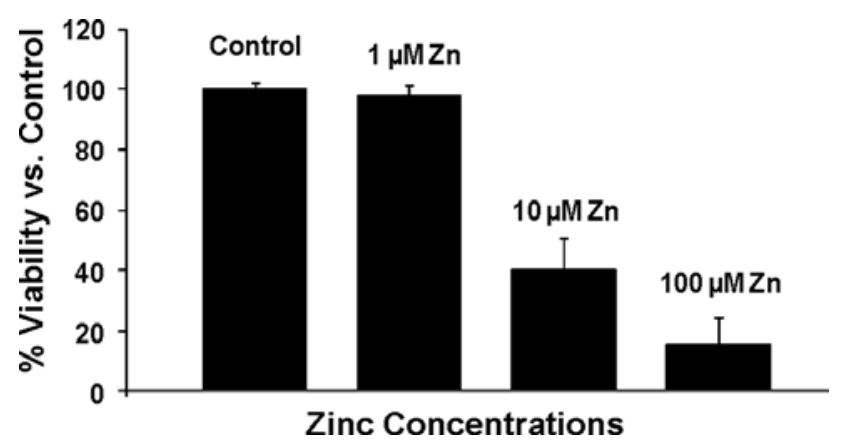

Figure 4. HEKa viability measured by MTT assays after zinc treatments. The graph of the percentage of MTT reduction after the treatment of zinc at 1,10 , and $100 \mu \mathrm{M}$ for 30 min (means $\% \pm \mathrm{SE}, \mathrm{n}=6$ samples per treatment).

study demonstrate a potentially important link between alteration in intracellular zinc homeostasis and apoptotic effects associated with UV-induced oxidative stress in HEKa.

$U V B$ radiation is known to be a potent inducer of significant amounts of reactive oxygen species (ROS) production, which have been implicated in skin inflammation and cancer. Though the exact mechanism behind UV-induced toxicity is yet to be elucidated, many studies have suggested oxidative stress playing an important role in UVB elicited DNA damage, cell membrane disruption and subsequent cell death (11-13,24-26). Several lines of evidence indicate that oxidative and nitrosative stress lead to an increase of intracellular zinc and zinc cytotoxicity that may, in turn, stimulate and interfere with signaling pathways (8-10,40,41). Zinc is normally tightly regulated inasmuch as intracellular concentrations of free zinc have been estimated to be in the pico- or nanomolar concentration range $(2,5,6)$. For example, levels of free zinc are several orders of magnitude less than that of calcium (42). A loss of this zinc homeostatic control causes cell injury or death via apoptosis or, in extreme circumstances, necrosis.

In this study, we demonstrated that UVB-mediated toxicity alters cellular zinc homeostasis. Given the fact that there is a well documented link between zinc accumulation and oxidative stress-induced cell death, there is a welldefined possibility that the release of zinc observed in this study is due to oxidative modification of zinc containing proteins. Among the intracellular proteins that maintain zinc at such low concentrations are the metallothionein (MT) proteins, capable of sequestering up to 7 zinc ions per monomer (1), and thus provide strong buffering capacity for cytosolic zinc. There is growing evidence suggesting that $\mathrm{Zn}^{2+}$ is released from the metalloproteins such as metallothioneins following oxidative stress (43-45).

Our data show that, after UVB irradiation, there is a substantial increase in intracellular zinc concentration. The increase in intracellular zinc is detected immediately after UVB irradiation, and shows a dose-dependent relationship with the irradiation. For example, the change of zinc fluorescence was observed after only $15 \mathrm{sec}\left(105 \mathrm{~mJ} / \mathrm{cm}^{2}\right)$ irradiation, and higher fluorescent intensity was measured after longer irradiation. The UVB dose-dependent increase appeared to reach a peak with $5 \mathrm{~min}\left(2,100 \mathrm{~mJ} / \mathrm{cm}^{2}\right)$ for Newport Green loaded cells, with $2 \mathrm{~min}\left(840 \mathrm{~mJ} / \mathrm{cm}^{2}\right)$ for FluoZin-3.
However, this may not be the highest intracellular zinc released by UVB as the fluorescent zinc detection is associated with the $K_{D}$ value or the range of a fluorescent indicator. UVA was also shown to produce increases in intracellular zinc when it was measured more than one hour after cells was irradiated $(46,47)$. In the present study the continual changes in zinc fluorescence were measured immediately before and after the irradiation. It remains to be seen if UVA radiation can also induce an immediate rise of zinc. Taken together, these observations are consistent with a model that oxidative processes release zinc from MT by redox regulation (29). Although the mechanism which accounts for the increase in cytoplasmic zinc is still elusive, the available data suggest that zinc release occurs via a ROS-mediated pathway $(11,40,41,43)$. Thus, ROS induced by UVB radiation may attribute to UV-induced rising zinc in HEKa through oxidative stress. Accumulating evidence indicates that zinc is also involved in oxidative cytotoxicity of other pathological conditions, such as ischemia/reperfusion, head trauma, Alzheimer's disease, endothelial cell death of cardiovascular disease, impaired immune responses, and hepatic oxidative stress $(7,8,41,45)$.

Interestingly, zinc itself is a strong inducer of oxidative stress by promoting mitochondrial and extra-mitochondrial production of reactive oxygen species (48). In contrast to the biological necessity of relatively lower concentrations of zinc, elevated levels of this ion has shown to be potently toxic to eukaryotic cells $(7,49)$. In the present study, we directly increased the intracellular zinc with the application of zinc and its ionophore, which induced a greater reduction in HEKa viability (Figs. 3 and 4). This observation is consistent with the literature concerning zinc toxicity (7).

Zinc was applied for only $30 \mathrm{~min}$ in the cytotoxicity tests of the present study. We project that prolonged incubation with zinc would induce greater cell injury/death. While the cellular mechanisms of zinc mediated apoptosis and cell injury is still elusive, we have noted the fact that the proposed cell death pathways triggered by intracellular free zinc are very similar to that induced by UVB radiation. For example, the accumulation of zinc (as well as UVB radiation) have been reported to activate the pro-apoptotic mechanisms such as 12LOX, ERK1/2 or p38 MAPK $(35,43,50,51)$. Zinc accumulation or dyshomeostasis is also reported to impair mitochondrial function leading to a loss of mitochondrial membrane potential $(\Delta \Psi \mathrm{m})$, as well as to increased generation of ROS and the mitochondrial apoptogenesis (37-39). Thus, the rising zinc observed in the present work could be a newly observed factor in UV-induced signaling pathways given its ability to amplify the production of ROS. UVB induced zinc dyshomeostasis and oxidative stress might act synergistically to promote phototoxicity. Thus, we need to explore further the possible contribution of crosstalk between ROS and zinc to UVB-induced apoptotic signal pathways.

Zinc also has direct antioxidant effects and is known to participate as cofactor of several antioxidative enzymes including superoxide dismutase (SOD) (52). Zinc deficiency certainly increases oxidative stress as well (53). It seems reasonable to assume that the action of zinc is concentrationdependent. While moderate increases protect cells from oxidative stress, higher amounts have the opposite effect. In 
the case of UVB-induced rising intracellular zinc, the initial increase of zinc may even be beneficial, perhaps with zinc acting as an antioxidant since it is required by several ROS scavangers such as $\mathrm{Cu} / \mathrm{Zn}$ SOD. However, continuous zinc increases are detrimental. While the mechanisms by which zinc mediates or prevents oxidative stress-induced injury are complex, cells are also expected to possess a specific zinc set-point $[\mathrm{pZn}$ as described in $(7,54)]$, by which too little or too much zinc can promote cellular demise. As such, the alteration of zinc homeostasis can upset the delicate balance by which zinc exerts its toxic or protective capabilities. Finally, oxidative cell death may be merely one of the consequences caused by zinc accumulation. As zinc is integral to the production of many functional proteins, and transcription factors, the disturbance of zinc homeostasis by UVB may have multifaceted effects such as DNA damage and the development of skin cancer, possibilities which merit further investigation.

\section{Acknowledgements}

This work was supported in part by a grant from The National Institutes of Health (Y.V.L).

\section{References}

1. Maret W and Sandstead HH: Zinc requirements and the risks and benefits of zinc supplementation. J Trace Elem Med Biol 20: 3-18, 2006.

2. Vallee BL and Falchuk KH: The biochemical basis of zinc physiology. Physiol Rev 73: 79-118, 1993.

3. Berg JM: Zinc fingers and other metal-binding domains Elements for interactions between macromolecules. J Biol Chem 265: 6513-6516, 1990.

4. Davis AM and Berg JM: Homodimerization and heterodimerization of minimal zinc(II)-binding-domain peptides of T-cell proteins CD4, CD8alpha, and Lck. J Am Chem Soc 131: 11492-11497, 2009.

5. Outten CE and O'Halloran TV: Femtomolar sensitivity of metalloregulatory proteins controlling zinc homeostasis. Science 292: 2488-2492, 2001.

6. Cousins RJ, Liuzzi JP and Lichten LA: Mammalian zinc transport, trafficking, and signals. J Biol Chem 281: 24085-24089, 2006.

7. Frederickson CJ, Koh JY and Bush AI: The neurobiology of zinc in health and disease. Nat Rev Neurosci 6: 449-462, 2005

8. Kroncke KD: Cellular stress and intracellular zinc dyshomeostasis. Arch Biochem Biophys 463: 183-187, 2007.

9. Mocchegiani E and Malavolta M: Zinc dyshomeostasis, ageing and neurodegeneration: implications of A2M and inflammatory gene polymorphisms. J Alzheimers Dis 12: 101-109, 2007.

10. Hao Q and Maret W: Aldehydes release zinc from proteins. A pathway from oxidative stress/lipid peroxidation to cellular functions of zinc. FEBS J 273: 4300-4310, 2006.

11. Wiswedel I, Keilhoff G, Dorner L, et al: UVB irradiation-induced impairment of keratinocytes and adaptive responses to oxidative stress. Free Radic Res 41: 1017-1027, 2007.

12. Lippens S, Hoste E, Vandenabeele P, Agostinis P and Declercq W: Cell death in the skin. Apoptosis 14: 549-569, 2009.

13. Noonan FP, Recio JA, Takayama H, et al: Neonatal sunburn and melanoma in mice. Nature 413: 271-272, 2001.

14. Bickers DR and Athar M: Oxidative stress in the pathogenesis of skin disease. J Invest Dermatol 126: 2565-2575, 2006.

15. Kumar B, Koul S, Khandrika L, Meacham RB and Koul HK: Oxidative stress is inherent in prostate cancer cells and is required for aggressive phenotype. Cancer Res 68: 1777-1785, 2008.

16. Wang K, Fang H, Xiao D, et al: Converting redox signaling to apoptotic activities by stress-responsive regulators HSF1 and NRF2 in fenretinide treated cancer cells. PLoS One 4: E7538, 2009.
17. Van Laethem A, Garmyn M and Agostinis P: Starting and propagating apoptotic signals in UVB irradiated keratinocytes. Photochem Photobiol Sci 8: 299-308, 2009.

18. Nishigori C: Cellular aspects of photocarcinogenesis. Photochem Photobiol Sci 5: 208-214, 2006.

19. Brenner M, Coelho SG, Beer JZ, et al: Long-lasting molecular changes in human skin after repetitive in situ UV irradiation. J Invest Dermatol 129: 1002-1011, 2009.

20. Brenner $M$ and Hearing VJ: The protective role of melanin against UV damage in human skin. Photochem Photobiol 84: 539-549, 2008.

21. Katiyar SK: UV-induced immune suppression and photocarcinogenesis: chemoprevention by dietary botanical agents. Cancer Lett 255: 1-11, 2007.

22. Alt A, Lammens K, Chiocchini C, et al: Bypass of DNA lesions generated during anticancer treatment with cisplatin by DNA polymerase eta. Science 318: 967-970, 2007.

23. Marrot L and Meunier JR: Skin DNA photodamage and its biological consequences. J Am Acad Dermatol 58: S139-S148, 2008.

24. Charruyer A, Bell SM, Kawano M, et al: Decreased ceramide transport protein (CERT) function alters sphingomyelin production following UVB irradiation. J Biol Chem 283: 16682-16692, 2008.

25. Perluigi M, Giorgi A, Blarzino C, et al: Proteomics analysis of protein expression and specific protein oxidation in human papillomavirus transformed keratinocytes upon UVB irradiation. J Cell Mol Med 13: 1809-1822, 2009.

26. Zhang Q, Southall MD, Mezsick SM, et al: Epidermal peroxisome proliferator-activated receptor gamma as a target for ultraviolet B radiation. J Biol Chem 280: 73-79, 2005.

27. Cortese-Krott MM, Munchow M, Pirev E, et al: Silver ions induce oxidative stress and intracellular zinc release in human skin fibroblasts. Free Radic Biol Med 47: 1570-1577, 2009.

28. Lee SJ, Cho KS and Koh JY: Oxidative injury triggers autophagy in astrocytes: the role of endogenous zinc. Glia 57: 1351-1361, 2009.

29. Maret W: Oxidative metal release from metallothionein via zinc-thiol/disulfide interchange. Proc Natl Acad Sci USA 91: 237-241, 1994.

30. Stork CJ and Li YV: Measuring cell viability with membrane impermeable zinc fluorescent indicator. J Neurosci Methods 155: 180-186, 2006.

31. Martin JL, Stork CJ and Li YV: Determining zinc with commonly used calcium and zinc fluorescent indicators, a question on calcium signals. Cell Calcium 40: 393-402, 2006.

32. Stork CJ and Li YV: Intracellular zinc elevation measured with a 'calcium-specific' indicator during ischemia and reperfusion in rat hippocampus: a question on calcium overload. J Neurosci 26: 10430-10437, 2006.

33. Canzoniero LM, Turetsky DM and Choi DW: Measurement of intracellular free zinc concentrations accompanying zinc-induced neuronal death. J Neurosci 19: RC31, 1999.

34. Colvin RA, Bush AI, Volitakis I, et al: Insights into $\mathrm{Zn}^{2+}$ homeostasis in neurons from experimental and modeling studies. Am J Physiol Cell Physiol 294: C726-C742, 2008.

35. Klein C, Creach K, Irintcheva V, et al: Zinc induces ERKdependent cell death through a specific Ras isoform. Apoptosis 11: 1933-1944, 2006.

36. Stork CJ and Li YV: Rising zinc: a significant cause of ischemic neuronal death in the CA1 region of rat hippocampus. J Cereb Blood Flow Metab 29: 1399-1408, 2009.

37. Sensi SL, Yin HZ and Weiss JH: AMPA/kainate receptortriggered $\mathrm{Zn} 2^{+}$entry into cortical neurons induces mitochondrial $\mathrm{Zn} 2^{+}$uptake and persistent mitochondrial dysfunction. Eur J Neurosci 12: 3813-3818, 2000.

38. Feng $\mathrm{P}$, Liang JY, Li TL, et al: Zinc induces mitochondria apoptogenesis in prostate cells. Mol Urol 4: 31-36, 2000.

39. Gazaryan IG, Krasinskaya IP, Kristal BS and Brown AM: Zinc irreversibly damages major enzymes of energy production and antioxidant defense prior to mitochondrial permeability transition. J Biol Chem 282: 24373-24380, 2007.

40. Wiseman DA, Sharma S and Black SM: Elevated zinc induces endothelial apoptosis via disruption of glutathione metabolism: role of the ADP translocator. Biometals 23: 19-30, 2009.

41. Wiseman DA, Wells SM, Hubbard M, Welker JE and Black SM: Alterations in zinc homeostasis underlie endothelial cell death induced by oxidative stress from acute exposure to hydrogen peroxide. Am J Physiol Lung Cell Mol Physiol 292: L165-L177, 2007. 
42. Finney LA and O'Halloran TV: Transition metal speciation in the cell: insights from the chemistry of metal ion receptors. Science 300: 931-936, 2003.

43. Bossy-Wetzel E, Talantova MV, Lee WD, et al: Crosstalk between nitric oxide and zinc pathways to neuronal cell death involving mitochondrial dysfunction and $\mathrm{p} 38$-activated $\mathrm{K}^{+}$ channels. Neuron 41: 351-365, 2004.

44. Cuajungco MP and Lees GJ: Zinc metabolism in the brain: relevance to human neurodegenerative disorders. Neurobiol Dis 4: 137-169, 1997.

45. St Croix CM, Wasserloos KJ, Dineley KE, Reynolds IJ, Levitan ES and Pitt BR: Nitric oxide-induced changes in intracellular zinc homeostasis are mediated by metallothionein/ thionein. Am J Physiol Lung Cell Mol Physiol 282: L185-L192, 2002.

46. Pirev E, Calles C, Schroeder P, Sies H and Kroncke KD: Ultraviolet-A irradiation but not ultraviolet-B or infrared-A irradiation leads to a disturbed zinc homeostasis in cells. Free Radic Biol Med 45: 86-91, 2008.

47. Lutter K, De Spirt S, Kock S, et al: 3,3'-Dihydroxyisorenieratene prevents UV-induced formation of reactive oxygen species and the release of protein-bound zinc ions in human skin fibroblasts. Mol Nutr Food Res 54: 285-291, 2009.
48. Frazzini V, Rockabrand E, Mocchegiani E and Sensi SL: Oxidative stress and brain aging: is zinc the link? Biogerontology 7: 307-314, 2006

49. Frederickson CJ and Bush AI: Synaptically released zinc: physiological functions and pathological effects. Biometals 14: 353-366, 2001

50. Park JA and Koh JY: Induction of an immediate early gene egr-1 by zinc through extracellular signal-regulated kinase activation in cortical culture: its role in zinc-induced neuronal death. J Neurochem 73: 450-456, 1999.

51. Zhang Y, Wang H, Li J, et al: Peroxynitrite-induced neuronal apoptosis is mediated by intracellular zinc release and 12lipoxygenase activation. J Neurosci 24: 10616-10627, 2004.

52. Prasad AS: Clinical, immunological, anti-inflammatory and antioxidant roles of zinc. Exp Gerontol 43: 370-377, 2008.

53. Powell SR: The antioxidant properties of zinc. J Nutr 130: S1447-S1454, 2000

54. Krezel A and Maret W: Zinc-buffering capacity of a eukaryotic cell at physiological pZn. J Biol Inorg Chem 11: 1049-1062, 2006. 